\section{Vaginal length after a laparoscopic sacropexy}

\author{
Kristina Drusany Starič, ${ }^{1}$ \\ Adolf Lukanović, ${ }^{1}$ Matija Barbič, ${ }^{1}$ \\ Mija Blaganje, ${ }^{1}$ Adriana Cvijić, \\ Igor But ${ }^{2}$
}

${ }^{1}$ Division of Obstetrics and Gynecology, Ljubljana University Medical Center, Ljubljana; ${ }^{2}$ Division of Gynecology and Perinatology, Maribor University Medical Center, Maribor, Slovenia

\begin{abstract}
Vaginal shortening after surgical treatment of pelvic organ prolapse is associated with dyspareunia, which negatively affects women's sexual life as well as their psychosocial well-being. The aim of the study is to determine the vaginal length in women with high-grade pelvic organ prolapse treated with laparoscopic sacropexy. In the prospective study we included 22 women with high-grade prolapse of the uterus or vagina that were treated. They underwent a gynaecological examination with a measurement of the vaginal length, as well as the evaluation of the degree of prolapse prior to the procedure (laparoscopic sacropexy). The second measurement and evaluation of the vaginal length during the follow-up examination between 6 to 12 weeks after surgery was done. The control group included 23 healthy women, without genital prolapse. There was no statistically significant difference in the mean vaginal length before and after surgery in the group of treated women.
\end{abstract}

\section{Introduction}

Pelvic organ prolapse affects about $30 \%$ of women of all age groups. ${ }^{1}$ The prolapse has a significant effect on the patient's quality of life. The assessment of the quality of life of patients is evaluated with a standardized questionnaire assessing the problems with urinary retention and difficulties passing stools, evaluation of sexual dysfunction and the impact of problems on the patient's everyday life and psychosocial health. ${ }^{2}$

There is an estimated risk that $11.1 \%$ of women in the USA will have to undergo prolapse or urinary incontinence surgery by the age of 80 years. $^{3}$ In the USA there is up to 300,000 surgeries each year due to pelvic organ prolapse (22.7/10,000 women) and 13 to $25 \%$ of reoperations due to relapses, which is also a major financial problem. ${ }^{4}$

Laparoscopic and robotic assisted sacropexy are newer procedures, which are technically more demanding and expensive, but on the other hand offer shorter hospitalization and shorter recovery time with the same results as open surgery. Laparoscopic sacropexy with a mesh is carried out in women with a vaginal vault prolapse after a hysterectomy and failed previous surgical procedures. A fixation of the uterus to the sacrum is possible in women who want to keep the uterus. ${ }^{5}$

Literature data show a good success rate of robotic assisted laparoscopic supracervical hysterectomy and sacropexy; however, additional monitoring of the results and performance evaluation of the new surgical technique for the treatment of an apical prolapse is required due to the small number of treated patients. With the laparoscopic supracervical hysterectomy and sacrocervicopexy there is a lower risk for perioperative complications and an expected higher satisfaction rate of the patients. ${ }^{6}$

The surgical treatment of pelvic organ prolapse or vaginal surgery for other reasons may result in shortening of the vagina, which is connected with dyspareunia. Dyspareunia is a sexual dysfunction defined as genital pain occurring before, during and after sexual intercourse, and is a common clinical problem. The cause may be organic, emotional or psychological. ${ }^{7}$ Dyspareunia is more common after vaginal hysterectomy than abdominal hysterectomy. ${ }^{8}$ The pain caused by tissue scarring or nerve damage eventually decreases or wears off. The pain usually occurs continuously in cases where the vagina is shortened and there is scar tissue on vaginal vault. ${ }^{9}$

In 1952, Jewett had already found that the vagina is reduced in approximately $10 \%$ of patients after a total abdominal hysterectomy ( 0.4 to $2.3 \mathrm{~cm}) .{ }^{10}$

In a study which included 3247 patients it was found that with a hysterectomy there was a shortening of the vagina for approximately $0.63 \mathrm{~cm}$. After reconstructive surgery of the pelvic floor (bladder suspension, anterior/posterior colporrhaphy, apical prolapse surgery and other procedures), the length of the vagina was shortened for about $0.22 \mathrm{~cm}$. The shortening of the vagina was approximately $0.38 \mathrm{~cm}$ in anterior/posterior colporrhaphy. A hysterectomy without an anterior/posterior colporrhaphy was associated with a lesser shortening of the vagina compared with a hysterectomy with an anterior/posterior colporrhaphy. The shortening of the vagina is, besides hysterectomy and other pelvic floor surgery, also influenced by age, menopause and the patient's constitution (height and weight). ${ }^{11}$
Correspondence: Kristina Drusany Starič, Division of Obstetrics and Gynecology, Ljubljana University Medical Center, Šlajmerjeva 3, 1000 Ljubljana, Slovenia.

Tel.: 0038615226025

E-mail: tinaobgyn@yahoo.com

Key words: Vaginal length; Laparoscopic sacral fixation; Dyspareunia.

Contributions: the authors contributed equally.

Conflict of interest: the authors declare no potential conflict of interest.

Funding: none.

Received for publication: 23 September 2018. Revision received: 21 November 2018 Accepted for publication: 21 March 2019

This work is licensed under a Creative Commons Attribution NonCommercial 4.0 License (CC BY-NC 4.0).

(C) Copyright K. Drusany Starič et al., 2019

Licensee PAGEPress, Italy

Urogynaecologia 2019; 31:226

doi:10.4081/uij.2019.226

A study that included 165 patients treated for prolapse or incontinence established that there is a statistically significant difference in vaginal length before $(10.8 \pm 1.4)$ and after a reconstructive surgery of the pelvic floor $(9.8 \pm 1.3 \mathrm{~cm})$; however, the difference was probably not clinically significant, because there was no established link between the women's symptoms and measurements of the vagina. The mean value of vaginal shortening after surgery was $1.0 \pm 1.8 \mathrm{~cm} .{ }^{9}$ Emphasis was placed on the importance of keeping the vaginal length and size of the introitus after surgery on the pelvic floor. It was also found that the sexual function and satisfaction with sex life has improved or was unchanged after prolapse or urinary incontinence surgery. Dyspareunia was most frequently present in patients after posterior colporrhaphy $(26 \%)$ and after Burch colposuspension with colporrhaphy (38\%). ${ }^{12}$ In a large epidemiological cross-sectional observational study which included 1,004 women, aged 18 to 83 years, who had come on a regular outpatient gynaecological examination, the mean vaginal length was $9.6 \pm 1.5 .{ }^{13}$

\section{Materials and Methods}

In the prospective study we included 22 women with high-grade prolapse of the uterus or vagina without urinary inconti- 
Table 1. Demographic characteristics of surgically treated patients and of control group 1.

\begin{tabular}{lccc} 
Surgically treated patients $(\mathrm{n}=22)$ & Mean value \pm SD & Control group $(\mathrm{n}=23)$ & Mean value \pm SD \\
Age & $57.7 \pm 9.6$ & Age & $55.1 \pm 8.5$ \\
BMI & $25.9 \pm 2.7$ & BMI & $24.0 \pm 1.7$ \\
\hline Parity & $2.0 \pm 0.7$ & Parity & $1.2 \pm 0.8$ \\
Menopause $(\mathrm{n}=16)$ & $50.6 \pm 2.3$ & Menopause $(\mathrm{n}=14)$ & $50.4 \pm 2.0$ \\
\hline
\end{tabular}

SD, standard deviation. BMI, body mass index.

nence that were treated at the Department of Gynaecology, Division of Obstetrics and Gynaecology, University Medical Centre Ljubljana and at the Department of Gynaecology and Gynaecologic Urology, Division of Gynaecology and Perinatology, University Medical Centre Maribor, from March 2014 to March 2015. The examination was a part of regular check-up, so the ethical approval was not needed. Both centres perform laparoscopic sacrohysteropexy, and sacrocolpopexy. There were two cases of sacrocervicopexy at the Department of Gynaecology in Ljubljana. In all patients a mesh and screws were used for the fixation. The patients underwent a gynaecological examination with a measurement of the vaginal length in centimetres (TVL), prior to the procedure and during the follow-up examination between 6 to 12 weeks after surgery.

The control group included 23 healthy women, about the same age as the treated women, without prolapse, who were seen in the gynaecological outpatient clinic for other reasons, mostly for screening PAP tests. We also took vaginal length measurements (TVL). Basic demographic data (age, body mass index, parity and onset of menopause) were obtained from the patient' charts. We used a t-test for the statistical analysis of vaginal lengths before and after surgery. Paired Sample Correlations test was used to establish a correlation between the variables. Pearson correlation coefficient was used between variables in both groups. As there the TVL was the only question, we didn't include the complete pelvic organ prolapse score of the patients. There was no coincidental surgery of recto or cystocoele.

\section{Results}

Prior to the procedure the patients had the grade of prolapse $\geq$ II according to the classification. The youngest treated patient was 36 years old and the oldest 72 years. The control group of examined women is a group similar in age but without prolapse (pelvic organ prolapse quantification grade

Table 2. Type of sacropexy performed.

Sacrohysteropexy Number of patients $(n=22)$

Sacrocolpopexy

$16(72.7 \%)$

Sacrocervicopexy

$4(18.2 \%)$

Supracervical hysterectomy and sacrocervicopexypexy

$1(4.5 \%)$

$1(4.5 \%)$

Table 3. Vaginal length in patients before and after surgery.

\begin{tabular}{lccc} 
Surgically treated patients & TVL before surgery & TVL after surgery & P value \\
Total $(\mathrm{n}=22)$ & $9.41 \pm 0.96$ & $9.55 \pm 0.89$ & 0.208 \\
Sacrohysteropexy $(\mathrm{n}=16)$ & $9.72 \pm 0.71$ & $9.78 \pm 0.84$ & 0.432 \\
\hline
\end{tabular}

TVL, total vaginal length.

0 ) and mean TVL of $9.46 \pm 0.66 \mathrm{~cm}$ ( 8.5 to $11 \mathrm{~cm})$, which was measured during regular examination (Table 1).

Laparoscopic sacrocolpopexy was performed in four patients $(18.2 \%)$ who have had previously performed total hysterectomies due to various reasons (uterus myomatosus, endometrial hyperplasia and uterine prolapse in two patients). One of the patients that underwent a laparoscopic sacropexy previously had two unsuccessful prolapse surgeries; a vaginal hysterectomy with an anterior and posterior colporrhaphy, which was followed by a sacrospinous ligament suspension of the vaginal vault (Richter's procedure). The patient's vaginal length prior to the surgery was $7 \mathrm{~cm}$.

In one patient $(4.5 \%)$ we simultaneously performed a supracervical hysterectomy and a sacrocervicopexy. One patient (4.5\%) had, except for a hysteropexy, a myomectomy, sterilisation, anti-incontinence surgery (MiniArc) and a posterior colporrhaphy. She wished to keep her uterus (Table 2).

There is no statistically significant difference between vaginal lengths before and after the laparoscopic sacropexy $(\mathrm{P}>0.05)$ (Table 3 ). When comparing vaginal lengths of treated patients without a hysterectomy $(n=16)$ and the controls $(n=23)$ we found no statistically significant difference $(\mathrm{P}=0.241)$.

The average duration of a surgery was 124 minutes. Patients were hospitalized for 2 to 4 days. There were no perioperative complications. During their first follow-up examination the patients did not report about possible onset of dyspareunia or urinary incontinence. In one patient $(4.5 \%)$, the procedure was evaluated as unsuccessful after 3 months.

\section{Discussion}

Vaginal lengths measured after a laparoscopic sacral fixation were slightly greater $(9.55 \pm 0.89)$ in comparison with the lengths prior to surgery $(9.41 \pm 0.96)$; however, the difference is not statistically significant $(\mathrm{P}>0.05)$. In one patient with two previous unsuccessful uterine prolapse surgeries (first, a vaginal hysterectomy with an anterior and posterior colporrhaphy; second, a sacrospinous ligament suspension of the vaginal vault) the vaginal length was shortened due to previous surgeries to $\mathrm{TVL}=7.0$ $\mathrm{cm}$. After a laparoscopic sacrocolpopexy the patient's TVL was elongated for $2 \mathrm{~cm}$. This outcome may be the result of a method of examination or differences in measurements between doctors. On the other hand, the possible cause for the prolonged vaginal length is the surgical technique in which it is important to maintain the length of the vagina, because increased vaginal stretching and consequential lengthening of the vagina leads to changes in anatomy and postoperative iatrogenic urinary inconti- 
nence. After sacrohysteropexies and sacrocervicopexy there were no changes in vaginal length, which is an expected result, since the uterus is fixed to the sacrum without a vaginal interference. There was also no significant difference between vaginal lengths in patients with laparoscopic sacrohysteropexy and the control group of healthy women $(\mathrm{P}>0.05)$.

In one patient $(4.5 \%)$, the sacrohysteropexy was evaluated as unsuccessful after 3 months. The results found in literature point to the fact of a higher percentage of relapses after surgeries other than a complete hysterectomy $(23.8 \%)$ when compared to those in which the uterus is removed $(6.7 \%){ }^{14}$ When deciding on whether to perform a hysterectomy we always take the patient's wish into account.

Although a surgical extension of an iatrogenic vaginal shortening (Davydov's laparoscopic procedure) can be carried out in extreme cases, which improves dyspareunia and thereby the sexual function, ${ }^{14}$ it is better use the surgical procedure that doesn't shorten vagina.

\section{Conclusions}

We found that there is no vaginal shortening after a laparoscopic sacropexy. The surgical treatment is an ideal choice for younger, sexually active women with a high-grade prolapse. The results are promising, but the final assessment of changes in the vaginal length after a laparoscopic sacral fixation requires further studies on a larger number of surgical patients, and longer follow up.

\section{References}

1. Bump RC, Norton PA. Epidemiology and natural history of pelvic floor dysfunction, Obstet Gynecol Clin North Am 1998;25:723-46.

2. Srikrishna S, Robinson D, Cardozo L, Cartwright R. Experiences and expectations of women with urogenital prolapse: a quantitative and qualitative exploration. BJOG 2008;115:1362-8.

3. Olsen AL, Smith VJ, Bergstrom JO, et al. Epidemiology of surgically managed pelvic organ prolapse and urinary incontinence. Obstet Gynecol 1997;89: 501-6.

4. Barber MD, Maher C. Epidemiology and outcome assessment of pelvic organ prolapse. Int Urogynecol J 2013;24: 1783-90.

5. Show RW, Luesley D, Monga A. Gynaecology fourth edition. United Kingdom: Churchill Livingstone Elsevier; 2011. pp 849-860.

6. Benson AD, Kramer BA, Wayament RO, Schwartz BF. Supracervical robotic-assisted laparoscopic sacrocolpopexy for pelvic organ prolapse. JSLS 2010;14:525-30.

7. Montserrat E, Montserrat P, Francisco C. De novo dyspareunia after pelvic organ prolapse surgery. Gynecol Surg 2010;7:217-25.

8. Abdelmonem AM. Vaginal length and incidence of dyspareunia after total abdominal versus vaginal hysterectomy. Eur J Obstet Gynecol Reprod Biol 2010;151:190-2.

9. De La Cruz JF, Myers EM, Geller EJ. Vaginal versus robotic hysterectomy and concomitant pelvic support surgery: a comparison of postop rative vaginal length and sexual function. J Minim Invasive Gynecol 2014;21:1010-4.

10. Jewett JG. Vaginal length and incidence of dyspareunia following total abdominal hysterectomy. Am J Obstet Gynecol 1952;63:400-7.

11. Tan JS, Lukacz ES, Manefee SA, et al. Determinants of vaginal length. Am J Obstet Gynecol 2006;195:1846-50.

12. Weber AM, Walters MD, Schover LR, Mitchinson A. Vaginal anatomy and sexual function. Obstet Gynecol 1995;86:946-9.

13. Swift S, Woodman P, O'Boyle A, et al. Pelvic Organ Support Study (POSST): The distribution, clinical definition, and epidemiologic condition of pelvic organ support defects. Am J Obstet Gynecol 2005;192:795-806.

14. Morarty CR, Mikos JR, Moore RD. Case report: surgically shortened vagina lengthened by laparoscopic Davydov procedure. Female Pelvic Med Reconstr Surg 2013;19:303-5. 